vol. $30-n^{\circ} 1 \mid 2014$

Mineurs en migration : enjeux juridiques, politiques et sociaux

\title{
Signorelli Amalia e Miranda Adelina, Pensare e ripensare le migrazioni
}

Camille Schmoll

\section{CpenEdition}

Journals

Édition électronique

URL : https://journals.openedition.org/remi/6713

DOI : $10.4000 /$ remi.6713

ISSN : $1777-5418$

Éditeur

Université de Poitiers

Édition imprimée

Date de publication : 1 mars 2014

Pagination : 157-159

ISBN : 979-10-90426-21-4

ISSN : 0765-0752

Référence électronique

Camille Schmoll, «Signorelli Amalia e Miranda Adelina, Pensare e ripensare le migrazioni », Revue européenne des migrations internationales [En ligne], vol. 30 - $\mathrm{n}^{\circ} 1$ | 2014, mis en ligne le 01 mars 2014, consulté le 14 avril 2022. URL : http://journals.openedition.org/remi/6713 ; DOI : https://doi.org/ 10.4000/remi.6713 


\section{Notes de lecture}

Raffaele, Poli

Le marché des footballeurs. Réseaux et circuits dans l'économie globale. - Berne :

Peter Lang, 2010. - $164 \mathrm{p}$.

ISBN : 978-3-03-430358-3

Paru en 2010, cet ouvrage est le prolongement de la thèse de doctorat soutenue par l'auteur à l'Université de Neuchâtel. II a pour objectif de comprendre les logiques de fonctionnement du marché du travail des footballeurs en Europe dans la seconde moitié des années 2000 en mobilisant les outils conceptuels de la géographie économique et ceux de la sociologie économique. L'analyse des réseaux et des circuits fournit à l'ouvrage sa trame centrale, jetant un pont entre ces deux spécialités. C'est en suivant ce fil conducteur que l'auteur tente de donner une intelligibilité aux déplacements des joueurs s'effectuant vers et à l'intérieur de I'UEFA. Pour ce faire, il mobilise une grande quantité de matériaux empiriques couvrant, le plus souvent, deux à trois décennies, ce qui lui permet de mettre en évidence les tendances lourdes structurant ce marché, mais aussi des changements plus récents, le tout étant agrémenté de nombreux tableaux et graphiques et de quelques cartes qui montrent comment des flux démographiques et économiques se projettent sur l'espace mondial.

Bâti en trois parties, l'ouvrage comporte une introduction qui rappelle les transformations dont le marché du travail des footballeurs est le produit. L'auteur en vient rapidement aux changements juridiques des années 1990, qui ont conduit à alléger les contraintes qui, depuis la fin de la Première Guerre mondiale, entravaient la circulation des joueurs entre les pays européens. Arrivé à ce stade, il énumère les conséquences économiques de ces changements juridiques, qui tiennent pour l'essentiel à une envolée du montant global des transactions commerciales, à un accroissement sans précédent des inégalités de richesse entre les ligues, les équipes et les joueurs et à la place cruciale qu'acquiert le marché des transferts dans l'économie des clubs de football. Ce sont ces inégalités, qui donnent lieu à des flux géographiquement ordonnés, que l'auteur tente d'expliquer dans cet ouvrage.

La première partie explicite le cadre théorique à l'intérieur duquel l'auteur déploie ses analyses. Se référant à Emirbayer, Raffaele Poli se réclame du relationnalisme, dont les principes empruntent au concept éliasien de configuration. Opposé au paradigme substantialiste qui conçoit le réel en tant que série d'entités isolées, existant à l'état figé de choses, le relationnisme privilégie une approche contextuelle, multilatérale et processuelle de la vie en société, assimilée à un maillage perpétuellement mouvant de réseaux d'acteurs qui se font et se défont au gré des affinités socialement sélectives qui conduisent les individus à se regrouper ou à s'éviter. À la suite de Granovetter, l'auteur met l'accent sur l'imbrication complexe des structures sociales, qui contraignent les comportements, et des actions des individus qui, en interagissant ensemble, contribuent à la formation de ces dernières tout en y trouvant des ressources permettant d'exercer du pouvoir sur autrui. $C^{\prime}$ est en adoptant cette perspective que l'auteur aborde la mondialisation. S'il reprend l'idée d'un vaste processus mû par des mobiles économiques, suscitant une reconfiguration territoriale des interdépendances sociales allant dans le sens du dépassement des entités étatiques, il n'adhère pas pour autant à l'hypothèse hyperglobaliste annonçant l'avènement d'un espace d'action homogène. L'espace 
mondialisé est, en fait, façonné par de multiples acteurs agissant en réseaux, qui, en s'affranchissant des frontières et des connexions spatiales historiquement héritées, jettent de nouveaux ponts entre territoires distants en fonction des opportunistes économiques qu'ils y détectent. C'est en ce sens que l'auteur insiste, d'une part, sur le rôle-clé joué par les intermédiaires dans la mobilité de la main-d'œuvre, eu égard au contrôle qu'ils exercent sur les canaux migratoires via leurs réseaux de relations, et, d'autre part, sur l'intérêt qu'ils ont à fractionner les espaces de production dans le but de créer des chaînes de valeur ajoutée.

La deuxième partie analyse la géographie du commerce international des footballeurs, ou plutôt des droits contractuels afférents, qui s'est déployée suite aux nouvelles facilités réglementaires et à l'essor des pratiques spéculatives pesant sur le marché des transferts. L'accentuation du statut de marchandise des footballeurs qui s'en est suivi est allée de pair avec une forte progression de la part de joueurs expatriés en Europe et, plus encore, dans les championnats et les équipes les mieux classés. En effet, dès lors que c'est l'ordre marchand qui assure la régulation des transferts, les performances sportives des ligues et des clubs sont très dépendantes de leurs capacités financières à recruter des joueurs issus des sélections nationales les plus cotées. À partir de là, l'auteur s'emploie à décrire la nouvelle division internationale du travail à l'œuvre dans le football marchand. II en fait la résultante d'un double processus, au principe de la création et du renforcement d'avantages ou de désavantages compétitifs : d'une part, l'élargissement considérable de l'espace de recrutement des joueurs à I'initiative des équipes européennes peu fortunées, contraintes de se tourner vers des championnats lointains où les transactions sont plus risquées, mais moins coûteuses, et d'autre part, la spécialisation dans la formation et l'exportation de joueurs de talent d'un nombre restreint de pays (Brésil, Argentine, France), qui sont les premiers à profiter de la croissance de la demande de travail sportif à l'échelle internationale. Les théories portant sur la division cognitive du travail, les rendements croissants ou les relations sociales localisées sont mobilisées pour rendre intelligibles les mécanismes de rétroaction qui assurent la pérennité de tels différentiels économiques entre territoires (Europe versus Afrique). Néanmoins, cet ordre inégalitaire ne se limite pas à la reproduction des structures spatiales établies. L'auteur montre que la géographie contemporaine du commerce international des footballeurs fait coexister des morphologies spatiales anciennes et une homogénéisation partielle des flux entre territoires, témoignant d'une diversification des circuits commerciaux.

L'analyse de la place centrale qu'occupent les intermédiaires dans les réseaux de transferts est au cœur de la dernière partie du livre. L'auteur conçoit la mondialisation du marché des joueurs comme une conséquence du déploiement planétaire des recruteurs, des agents de joueurs et des observateurs qui forment ensemble une multitude de toiles relationnelles, plus ou moins interconnectées, couvrant des aires géographiques d'étendue très variée. II décrit tout particulièrement les stratégies différenciées des agents en fonction de leur taille et de leur rayonnement. II s'attarde ensuite sur le pouvoir qu'ils exercent sur les autres acteurs. II souligne, tout d'abord, les connivences qu'ils établissent avec les entraîneurs et dirigeants, au risque de graves conflits d'intérêts voire de malversations financières, ce qui leur assure une rente relationnelle leur permettant d'accroître l'emprise qu'ils ont sur les flux de transfert. Puis, il décrit la forte dépendance dans laquelle sont souvent placés les joueurs expatriés à leur égard, ce qui l'amène à discuter du désastre humain auquel aboutit ce système spéculatif, de nombreux joueurs migrants, du fait de leur grande vulnérabilité, faisant l'objet d'une exploitation économique effrénée, quand 
il ne s'agit pas de trafics d'êtres humains.

Au final, cet ouvrage apporte des connaissances originales sur la manière dont l'activité économique et les structures territoriales liées au football sont encastrées dans de vastes réseaux de relations. Toutefois, on regrettera qu'il se limite parfois à une analyse balistique des flux migratoires, faisant l'impasse sur les significations que revêt la mobilité aux yeux des joueurs, ce que l'auteur avait pourtant commencé à traiter dans des travaux antérieurs.

Claude Lafabrègue

Maître de Conférences Université de Caen Basse-Normandie

\section{Andrei, Korliarov \\ Culture russe en exil, Europe 1917-1947. - Paris : YMCA-PRESS, 2013. $-720 \mathrm{p}$. \\ ISBN : 978-2-85065-275-2}

L'historien et iconographe Andrei Korliakov offre, avec son nouvel ouvrage, Culture russe en exil, Europe 1917-1947, un récit émouvant, destiné à tous ceux qui s'intéressent à l'histoire de l'émigration russe en Europe dans l'entre-deuxguerres. Cet album de photographies thématiques comprend 2100 images, qui s'étalent sur les 720 pages de l'ouvrage, un ample reportage dont l'originalité est de proposer au lecteur des clichés rares, collectés pendant plus de dix années et encore jamais révélés au public.

Dans Culture russe en exil, dont l'objectif est d'évoquer tous ceux qui contribuèrent à conserver et à développer en émigration leur culture d'origine, Andrei Korliakov raconte le parcours des artistes de I'ancien Empire russe, vedettes, mais aussi amateurs, depuis les premiers jours de leur exil. De Gallipoli et Constantinople à la France, en passant par la Bulgarie, la Serbie, la Tchécoslovaquie, l'Allemagne, les pays baltes, l'Angleterre, la Belgique, I'Espagne et I'Italie. Plus de 1100 exilés sont cités. Ce faisant, I'auteur dévoile, avec beaucoup de précision historique dans les commentaires accompagnant chaque image, le quotidien de ces Russes, qu'il laisse le lecteur découvrir et redécouvrir le long des huit chapitres de l'ouvrage que sont les volets consacrés à l'art de la photographie, au théâtre, au cinéma, à la musique et à la danse, à la littérature, aux arts plastiques, aux musées russes en exil et aux Journées de la culture russe.

Par sa démarche, Andrei Korliakov donne tout d'abord la parole à la photographie et aux photographes russes en exil, domaine jusqu'à présent peu étudié, mettant en lumière les noms aujourd'hui oubliés de Choumoff, doyen des photographes d'art russe à Paris, Brodsky, Markovitch, Lipnitzki et beaucoup d'autres, spécialisés dans le reportage et la photographie artistiques. On rencontre les photographes civils, mais aussi les militaires, par exemple Kritsky, lieutenant de la garde impériale du régiment Volynsky, évacué en 1920 à Gallipoli, où il rassembla une collection de plusieurs milliers de photographies, dispersées ensuite dans le monde entier. Outre la vie quotidienne du campement, les clichés des photographes militaires immortalisèrent de nombreuses scènes de loisirs comme les expositions et les autres prestations artistiques.

Bien qu'entamant une réflexion complexe sur l'impact de la culture sur la vie en émigration, ces photographies servent aussi d'ouverture au grand volet consacré au théâtre russe en exil, où on voit défiler les portraits des dramaturges, des metteurs en scène et des comédiens. Sur le chemin vers Constantinople, ils passèrent par le camp de Limassol (Chypre), par laTurquie et la Bulgarie, d'où ils repartirent pour l'Allemagne ou Paris. Par exemple, Duvan-Torzoff commença à travailler auThéâtre populaire à Sofia, puis s'engagea à l'Oiseau bleu à Berlin et dans le Groupe de Prague du Théâtre d'art de Moscou. Installé à Paris à partir de 1925, il se produisait au théâtre de l'Atelier chez Charles Dullin. 
Parlant du cinéma russe en exil, Andrei Korliakov évoque, à travers ces photographies, presque tous les films à sujet russe tournés dans l'entre-deux-guerres en Turquie, en Pologne, en Allemagne, en Italie et, bien évidemment, en France. II expose les photos de tournage, de décors, les cadres de films, les portraits des comédiens, des réalisateurs, des caméramans et des costumiers comme celles du célèbre peintre décorateur de l'époque Bilinsky. L'auteur conte ensuite les arts plastiques - sculpteurs, architectes, peintres et peintres d'icônes. Dès les premiers jours de l'exil, ils se mirent à organiser des expositions, d'abord dans les milieux des forces armées au camp de Gallipoli, ensuite pour un public plus vaste dans la partie européenne de la capitale turque. II en fut de même en Finlande et en Estonie, où furent évacuées des unités de l'armée du Nord-Ouest, ou encore en Allemagne, où se retrouvèrent des dizaines de milliers de réfugiés russes qui ont fondé leurs colonies, et en France, à Montparnasse. Ce chapitre débute avec des photos rarissimes signées Repine.

Traitant de la musique russe en exil, Andrei Korliakov dévoile devant son lecteur un panorama photographique des orchestres militaires, des chorales populaires, professionnelles et liturgiques. II montre aussi les chanteurs d'opéra, populaires et tziganes, des orchestres classiques ou de balalaïkas jusqu'aux grands compositeurs et artistes de l'opéra russe. Ensuite, il passe tout naturellement au ballet et aux danses populaires russes. De Diaghilev à Lifar, qui ont, chacun à leur manière, réalisé un exploit dans le domaine de la diffusion de l'art du ballet russe, et qui, dans leur art, furent secondés par d'autres artistes comme Preobragenskaya, Legat et Egorova.

Le chapitre consacré à la littérature englobe tous les aspects de l'activité littéraire, des écrivains militaires et des historiens (Tourkoul, Loukomsky, Solonevitch, etc.) au Prix Nobel Bounine, en passant par des ateliers d'impression et des maisons d'édition, par la presse russophone, les librairies et les bibliothèques. Comme celles des Maisons russes, de l'Université populaire ou encore du Zemgor. Enfin, le huitième et dernier chapitre développe le thème de la conservation et de la diffusion de la culture russe en émigration grâce aux Journées de la culture russe, aux fêtes et expositions Pouchkine ou encore grâce aux nombreux cercles, écoles et associations (les Sokols, les Vitiaz, I'Union des défenseurs de la pureté de la langue russe, etc.) et aux musées - musées militaires (musée des Cosaques, musée de la Cavalerie, etc.) et musées duThéâtre russe et de la Culture.

Outre les photographies - restaurées, mais pas retouchées -, Andrei Korliakov propose de nombreuses coupures de presse et affiches, des programmes des événements culturels et des catalogues des expositions, des cartes de visite, des cartes postales, des négatifs, qui complètent naturellement son récit photographique. Récit unique en son genre, car c'est la première fois qu'une communauté étrangère devient l'objet de la publication de tant de documents visuels thématiques, regroupés dans un seul recueil.

Culture russe en exil, Europe 1917-1947 est le cinquième et le plus gros volume dans la série L'émigration russe en photos, Europe 1917-1947, qui englobe déjà les albums Le Grand Exode russe, Europe 1917-1939 (2009, 1650 photos), Vers le succès, 1917-1947 (2005, 1000 photos), Honneur et dignité maintenus, 1917-1947 (2001, 750 photos) et Histoire illustrée de l'émigration russe, 1917-1947 (1999, 250 photos).

Kateryna Lobodenko-Senani Doctorante en art et médias Université Paris-III La Sorbonne nouvelle 
Amalia, Signorelli

Adelina, Miranda

Pensare e ripensare le migrazioni. - Palermo: Sellerio, 2011. $-328 \mathrm{p}$.

ISBN : 978-8-83892-559-7

On peut penser que le titre de cet imposant ouvrage, coordonné par Adelina Miranda et Amalia Signorelli, est un clin d'œil à celui de Nancy Green, Repenser les migrations, paru en 2002, qui rappelait combien la comparaison, diachronique et synchronique, est fondamentale à la mise en évidence des spécificités et des récurrences des situations migratoires. Adelina Miranda et Amalia Signorelli s'engagent dans cette direction en croisant une double attention aux flux d'immigration et d'émigration dans une perspective de long terme.

Issu d'un colloque organisé en décembre 2007 à l'Université de Naples Federico II, Pensare e ripensare le migrazioni nous livre de nombreuses clefs pour la compréhension des migrations, et pour interroger ce que l'on nomme fréquemment les " nouvelles formes migratoires ". II se compose de quatre parties thématiques, chacune suivie d'une discussion générale. Le titre annonce un objectif ambitieux. En réalité la réflexion proposée ne se base pas uniquement sur des textes d'ordre général et conceptuel, mais aussi sur des exemples et des études de cas, inspirés le plus souvent du contexte italien. De ce point de vue l'ouvrage apporte de nombreux éléments de connaissance empirique. II convoque certains sujets longtemps négligés des études migratoires traditionnelles, tels que la place et le rôle des femmes dans les différentes vagues d'émigration italienne à l'époque des migrations de masse (Andreina de Clementi) ; le traitement cinématographique des migrations (Matteo Sanfilippo) ; I'usage de la photographie par les émigrants italiens (Alberto Baldi).

\section{Migrations, inégalités, rapports de pouvoir}

Les auteurs des textes, essentiellement des anthropologues, des sociologues et des historiens, partagent une certaine défiance vis-à-vis du culturalisme et une attention pour les processus de stratification sociale et les formes de pouvoir qui contribuent à structurer les dynamiques migratoires. On retrouvera, entre autres, dans cet ouvrage, l'approche de sociologie historique de René Galissot, attentive aux relations entre culturalisme et dynamiques d'exploitation en contexte colonial et postcolonial ; la question de la mixité culturelle évoquée dans une perspective marxiste par Antonio Buttita ; I'attention d'Amalia Signorelli aux relations entre production de l'altérité et dynamiques de stratification sociale ; ou encore les déséquilibres Nord-Sud provoqués par les politiques migratoires du Nord évoqués par MarieAntoinette Hily et William Berthomière en conclusion. Toujours avec cette attention pour la production d'inégalités, Paola Corti montre, dans une analyse de longue durée de l'émigration italienne, comment celle-ci connaît à nouveau un processus de polarisation dans les dernières décennies, avec le renforcement de la fuite des cerceaux et, en parallèle, une émigration italienne très précaire trouvant des débouchés dans les segments les plus désavantagés des marchés du travail des pays d'accueil. Paola Corti met en évidence, loin du cliché du migrant circulant talentueux et élitaire, la complexité sociale des migrations italiennes contemporaines, ainsi que les nombreux mécanismes de précarisation et de déclassement qui peuvent se produire en migration.

Le texte de Carla Pasquinelli, consacré à la place de la question des mutilations génitales féminines dans la sphère publique, est passionnant pourcomprendre comment différents rapports de pouvoir se croisent dans l'émergence d'une controverse " culturelle ». Carla Pasquinelli relate les polémiques et réactions qui ont suivi la 
proposition d'introduction par un médecin basé en Toscane d'une pratique symbolique alternative à l'infibulation, qui lui avait été réclamée par un groupe de migrantes somaliennes, travailleuses domestiques pour la plupart. Une élite somalienne bien intégrée, installée depuis plus longtemps que ces migrantes en Italie, s'est coalisée avec des ONG et une partie de la classe politique pour se prononcer contre une telle pratique. Leur voix a été beaucoup plus relayée par les médias que celles des autres femmes. Cette controverse met en évidence la segmentation sociale au sein de la migration somalienne en termes de classe et de génération d'arrivée en Italie. Le récit livré par Carla Pasquinelli est édifiant, car il montre comment de complexes dynamiques de négociation interculturelle peuvent être traversées par différents rapports de pouvoir et comment des coalitions liées à une appartenance commune de classe sociale peuvent se mettre en place autour d'une pratique dite " culturelle " au-delà des différences ethniques, contribuant au final à définir comme "Autre " les plus faibles parmi les migrants.

\section{Transnationalisme et migrations féminines : de nouvelles formes migratoires?}

Comme dans tout débat sur les nouvelles formes migratoires, le transnationalisme joue un rôle très important dans cet ouvrage. Les auteurs ne sont pas nécessairement d'accord sur la nouveauté du phénomène, et l'on retrouvera les différentes positions du débat international, certains considérant, comme Paola Corti, que l'étude du transnationalisme correspond davantage à une évolution du regard des chercheurs, d'autres comme Sebastiano Ceschi essayant de décrypter la spécificité de la condition transnationale actuelle. On peut aussi remarquer la présence dans cet ouvrage d'analyses déjà fort connues du lecteur francophone, mais utilement traduites en italien comme celle d'Alain Tarrius, qui livre une synthèse efficace de ses travaux sur les nouvelles formes migratoires transnationales et les territoires circulatoires.

Une exploration des nouvelles formes migratoires $\mathrm{n}^{\prime}$ aurait pas été complète sans une réflexion sur la féminisation des migrations, à laquelle l'ouvrage consacre une importante partie. La part des femmes dans les migrations a-t-elle réellement augmenté ? Comment expliquer l'attention renouvelée des chercheurs pour le rôle des femmes? Les auteures de la partie consacrée aux femmes et à l'approche par le genre concordent pour dire que ce n'est pas tant la part des femmes dans les flux qui a augmenté, mais bien son rôle et sa capacité à se déplacer seule, indépendamment du regroupement familial. En parallèle c'est le regard que les chercheurs portent sur ces femmes qui a évolué. Andreina de Clementi restitue ainsi de façon passionnante le rôle central - mis en lumière par l'historiographie récente des femmes dans la grande émigration italienne vers les Amériques et dans la migration d'après-guerre vers l'Europe.

À partir de ses propres travaux et d'une revue impressionnante de la littérature, Mirjana Morokvasic évoque la question de la migration féminine comme parcours d'autonomisation. Elle montre comment les migrantes, sans cesse à la recherche de compromis plutôt que dans la rupture, utilisent l'ordre genré traditionnel pour poursuivre leurs propres objectifs quand elles ne peuvent pas directement le remettre en cause. Dans un contexte où la mobilité est devenue un important facteur de stratification sociale, la circulation apparaît comme une ressource importante pour nombre de ces femmes.

Comme on l'aura compris, l'ouvrage Pensare e ripensare le migrazioni est d'une grande richesse en ce qu'il se penche sur différentes époques et différents types de flux, tout en faisant preuve d'une profonde réflexivité sur l'évolution de la recherche sur les migrations. On retrouve cette 
attention pour les conditions de production de la connaissance sur les migrations dans le texte de Matteo Sanfilippo qui, tout en restituant les principaux courants de I'histoire de l'émigration italienne, contextualise son étude dans le cadre du débat historiographique qui l'a accompagné.

Pour finir, cet ouvrage a le mérite de proposer des textes en italien d'auteurs incontournables de la littérature francophone sur les migrations (voir les textes de Mirjana Morokvasic et Alain Tarrius, ainsi que les débats sur la circulation migra- toire présentés en conclusion par William Berthomière et Marie-Antoinette Hily). On espère, à l'inverse, que certains chapitres pourront être traduits en français, car ils apportent résolument du neuf au débat sur les nouvelles formes migratoires.

\section{..̊̊ Référence bibliographique}

Green Nancy (2002) Repenser les migrations, Paris, PUF, $139 \mathrm{p}$.

Camille Schmoll

Maître de Conférences

Université Paris 7 Denis Diderot/Laboratoire CNRS Géographie-cités 